\title{
WIDE DYNAMIC RANGE MICROELECTROMECHANICAL VISCOSITY SENSOR
}

\author{
Robert L. Borwick III, Philip A. Stupar, and Jeffrey F. DeNatale \\ Rockwell Scientific Company \\ Thousand Oaks, CA, USA
}

\begin{abstract}
A novel MEMS device has been designed to directly measure the viscosity of a fluid. The device, which incorporates both actuation and position sensing, is submerged in a fluid and measures the resistance to motion due to the viscous drag forces of the fluid. When this device is operated in the intended overdamped $(\xi>>1)$ regime, it has a response time that is linear with viscosity. Using our design, a single device is capable of measuring viscosity from $0.5 \mathrm{cP}$ to over $1000 \mathrm{cP}$ while maintaining a highly linear response. This same device also has good sensitivity; it can to detect viscosity changes on the order of $\pm 0.5 \%$.
\end{abstract}

\section{INTRODUCTION}

MicroElectromechanical (MEM) devices offer numerous advantages in the field of sensors. Their mechanical operation is inherently suited to the measurement of physical properties. Their compact size and portability can enable in-situ monitoring in both laboratory and field environments, providing valuable real-time information on system environment and health. One application that effectively exploits these benefits is a MEMS-based viscosity sensor [1-3].

The ability to accurately and efficiently assess the health and remaining useful life of lubricating fluids represents a critical element in implementing optimal condition based maintenance of machinery. Chemical and physical properties such as total acid number (TAN), $\mathrm{pH}$, water concentration, anti-oxidant concentration, particle size, and viscosity are among some of the properties used to evaluate the remaining useful life of lubricating fluids. Considerable effort over the years has been put into the development of chemical diagnostics for lubricating fluids, although sensors to provide a direct measure of fluid viscosity are less well developed. This paper presents the design, fabrication and experimental results of a MEMS viscosity sensor with high measurement linearity and wide dynamic range.

\section{THEORY OF OPERATION}

The sensor operates submerged in a fluid and senses the resistance to actuation due to the viscous drag forces of the fluid. The device is actuated and the time response of motion measured to directly determine the viscosity. We have designed the device to be over-damped when actuated in fluids of interest. Solving the equation of motion for an over-damped system [4], and assuming that we are sufficiently over-damped, the position as a function of time is given by:

$$
x(t)=x(o) e^{\left(\left(\zeta^{2}-1\right)^{1 / 2}-\zeta\right) \omega_{n} t}
$$

where $t$ is time, $\zeta$ is the damping coefficient, and $\omega_{\mathrm{n}}$ is the resonant frequency. This expression defines the final resting position as zero and the initial position as $x(0)$. If we define the response time as the time required to move a constant percentage of the initial displacement, we can divide equation 1 by the initial displacement and solve for the response time:

$$
t=C\left(\left(\zeta^{2}-1\right)^{1 / 2}+\zeta\right)
$$

Where $C$ is a constant that is a function of the displacement percentage and resonant frequency of the device. When this device is operated in the intended over-damped $(\zeta>>1)$ regime, the response time reduces to $t=2 C \zeta$ and is linear with the damping coefficient $\zeta$.

Additionally, the device has been designed to place a predominantly shear force on the fluid, and minimize the contributions of compressive interactions. Inter-digitated comb fingers on the device provide the shear on the fluid (fig. 1). Because the spacing between the comb fingers is small compared to the height and length, we can assume a linear velocity distribution in the liquid between the comb fingers, and therefore, the damping coefficient will be linearly proportional to the absolute viscosity of the fluid. These comb fingers are also used to capacitively measure the time response of the device motion.

All the measurements presented here were made on Newtonian fluids. However, this device is capable of measuring the viscosity of non-Newtonian fluids at different shear rates. Because the response time is not a function of total displacement, only the percentage of the initial displacement, different shear rates can be applied by changing the initial displacement. Therefore, we can measure the shear rate dependence of the viscosity of nonNewtonian fluids.

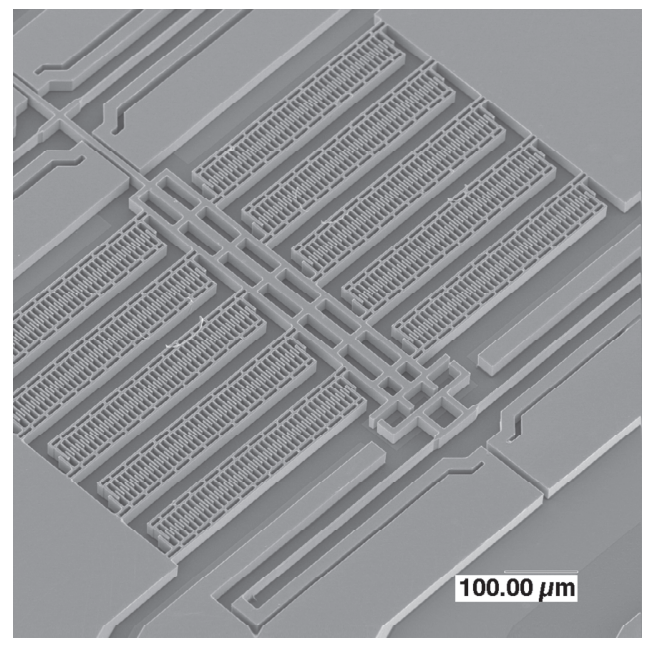

Figure 1. Micrograph of MEMS viscosity sensor. The comb fingers are long relative to their displacement to minimize fluid compression.

\section{DESIGN AND FABRICATION}

We have designed and fabricated two device types that are over-damped in a fluid that has a viscosity greater that $0.5 \mathrm{cp}$. Each device has an actuator and a capacitor for position sensing. These 
components are electrically isolated, but mechanically coupled using a $\mathrm{SiO}_{2}$ bridge. The primary difference between two device designs is the actuator. The two actuation methods that were explored offer distinct trade-offs in device operation. The actuation methods utilize a Lorentz force or an electrostatic force.

The Lorentz-force actuator utilizes the passing of a current, in the presence of a permanent magnetic field. For the present experiments, these are nominally $10 \mathrm{~mA}$ and $0.2 \mathrm{~T}$, respectively. This actuation method has the advantage of supplying a force that is independent of the dielectric properties of the fluid in which the viscosity sensor is submerged. This is a distinct advantage over an electrostatic drive, since the force applied will not change when measuring fluids with different dielectric constants nor will polar fluids affect it. However, having a permanent magnet in the package may be undesirable. Since many of the systems that would benefit from continuous monitoring of the viscosity have motors, there may be an abundance of ferrous particulates that would be attracted to the permanent magnet and may clog the sensor. However, utilizing a filter or device lid with a second permanent magnet to preferentially attract any ferrous particulates could mitigate this problem.

Electrostatic-force actuation does not require a permanent magnet to be present in the package, and a force comparable to the Lorentz force actuator is obtained with DC voltages on the order of 10 volts in a fluid with a dielectric constant of 2 . Simply adjusting the drive voltage can compensate for the issue of measuring multiple fluids with dramatically different dielectric constants. Because the response time is independent of the total displacement, small changes in the actuation force do not affect the device performance. However, the actuation voltage would need to be adjusted for a large dielectric change to ensure that the actuator does not hit the built in mechanical stops. Solving the issue of operation in polar fluids is more difficult and requires more complexity in the actuation method. By using an AC drive voltage that is faster than the response time of the polar fluid it is possible to use electrostatic actuation to measure viscosities of polar fluids [5].

The final required component of the device is the ability to sense position with respect to time. When a force is applied to the device, the position of the differential comb capacitor is measured using a commercially available capacitive sensing ASIC. This chip uses a $100 \mathrm{kHz}$ clock frequency to measure capacitance. This frequency has proven to be high enough to measure viscosities of polar fluids, including alcohols such as isopropanol. The interdigitated comb fingers used to capacitively measure the time response of the device motion also provide the shear on the fluid.

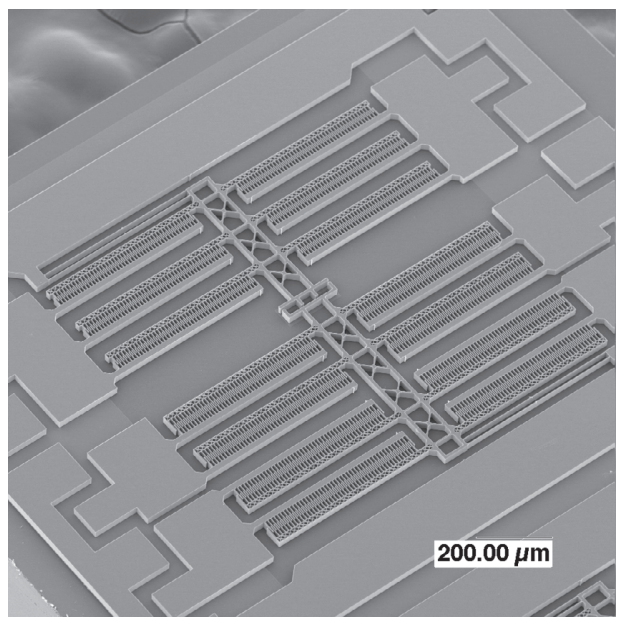

Figure 2. Micrograph of the electrostatic MEMS viscosity sensor.
The device is fabricated using the Rockwell Scientific aMEMS process [6], an SOI device layer transfer process. It consists of a $20 \mu \mathrm{m}$ silicon device layer that is patterned using DRIE to create the capacitive sensing comb structures and the device suspension. For the Lorentz-force actuator (fig. 1), the suspension doubles as the current carrying beam creating a force that results in a lateral deflection of the suspension. Coupled differential comb finger banks are used to create the capacitance change. In the case of the electrostatic actuator (fig. 2), the suspension provides a path to charge one half of the capacitor banks, while the other half is used for sensing the capacitance change. Here again the DRIE is used to form the drive capacitor bank.

\section{EXPERIMENT AND RESULTS}

Using both the Lorentz-force and electrostatic actuated devices, several measurements have been made. The majority of the results presented here will focus on the Lorentz-force actuator, however the functionality and linearity of the electrostatic drive has been verified. The following experimental results show the response time versus viscosity performance, the broad sensing range with excellent linearity, the device resolution and sensitivity, and finally a demonstration of the ability to distinguish fresh lubricant from degraded lubricant.

Before the sensors were tested, each one was affixed to a 12pin package with an inner cavity of $3.8 \mathrm{~mm} \times 4.8 \mathrm{~mm}$ and depth of $1.3 \mathrm{~mm}$. The actuator and sense capacitors were wire bonded, and the package was then mounted to a test board (fig. 3). The test board is shaped like a $\mathrm{T}$ to accommodate the signal I/Os and the capacitive sensing ASIC at one end, and the viscosity sensor at the opposing end. This configuration allows for a single drop of fluid to be placed in the package for testing, as well as completely submerging the sensor end of the board in a beaker of fluid. The single drop volume required to make a measurement is on the order of $20 \mu \mathrm{L}$. When the test board is submerged in a beaker of lubricant, a stir bar is used to circulate the fluid. The fluid flow in the beaker appears to have no measurable affect on the response time.

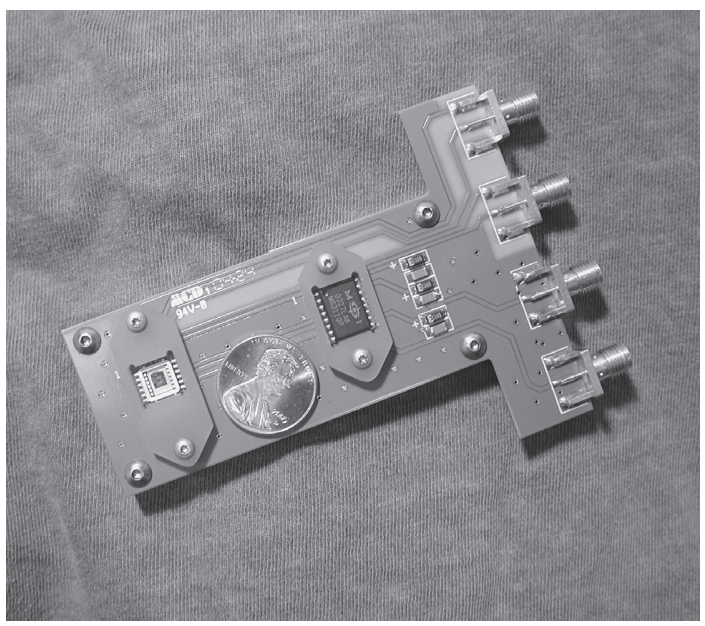

Figure 3. MEMS viscosity sensor mounted to a test board with capacitive sensing ASIC.

Calibrated viscosity standards were used to evaluate the viscosity sensor. These standards are silicone based and were chosen to have a range from 5 to $500 \mathrm{cp}$. Once a calibration factor was obtained from the viscosity standards, additional laboratory fluids were tested. These fluids included alcohols, such as 
propanol, octanol and decanol and alkanes such as heptane. Heptane was also used to clean the sensor in between tests. The silicone based viscosity standards are soluble in heptane and were easily removed by a light rinse. Once a device was cleaned it could be placed in a new fluid. There were no stiction problems observed if the device dried out completely. The only issue noted was that high viscosity fluids would take a little longer to re-wet a dry device. Re-wetting with heptane prior to a new measurement alleviates this issue.

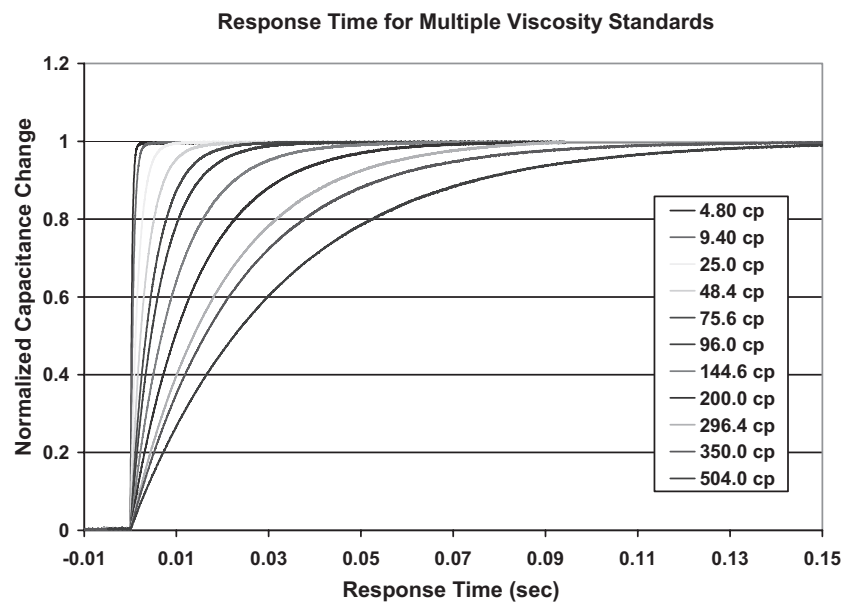

Figure 4. Response time traces for multiple calibrated viscosity standards.

By measuring the capacitance versus time for a step input current, the response time of the device, defined as the time required to move from $10 \%$ to $90 \%$ of the resting location, can be determined. As the viscosity of the fluid increases, the response time of the device also increases (fig. 4). The lower viscosity fluids $(0.5 \mathrm{cp})$ had a response time of less than one millisecond while the higher viscosity fluids $(1000 \mathrm{cp})$ required up to $200 \mathrm{~ms}$. The response time is related to the resonant frequency of the device and can be adjusted to suit the particular viscosity range that is being measured. A device designed to monitor a lubricant over a smaller viscosity range can be designed to have a sub millisecond response time, thereby allowing over 1000 measurements a second to be taken and averaged for noise reduction. Plotting the measured response time versus the viscosity of the fluid shows the highly linear response of the device over a wide range of viscosities spanning greater than three orders of magnitude (fig. 5). Resonant type viscosity sensors tend to have a limited linear range, and cannot resolve high viscosity fluids because the under-damped condition no longer holds [1]. Using our design, a single device is capable of measuring viscosity from $0.5 \mathrm{cP}$ to over $1000 \mathrm{cP}$ while maintaining a highly linear response.

The viscosity sensor also has good sensitivity, being able to detect viscosity changes on the order of $2 \%$. To test the sensitivity of the device, viscosity standards were mixed together to produce four solutions with $1 \%$ increments in viscosity (93-96cp). Multiple measurements were made in each of the four solutions (fig. 6). As shown, only one measurement point intersected with a neighboring viscosity measurement and viscosity changes of $2 \%$ are easily resolvable. This performance is comparable to large, rotary style viscometers such as those available from Brookfield Engineering [7].

After establishing the device linearity and resolution, a continuous measurement of viscosity was made to acquire repeatability and short-term reliability data. An overnight test in
Castrol 5050 was conducted, and a change of approximately 5\% was observed. This change was a larger than expected and did not appear to be random. We speculated that the change may have been temperature related, and remade the measurement over a 19 hour period while simultaneously observing the temperature of the fluid. The results of this experiment are seen in figure 7. The observed drift in viscosity was not due to sensor error, but was correlated with temperature changes of the fluid due to small variations in ambient temperature. For less than a 2 degree centigrade change, the fluid had a $5 \%$ viscosity change.

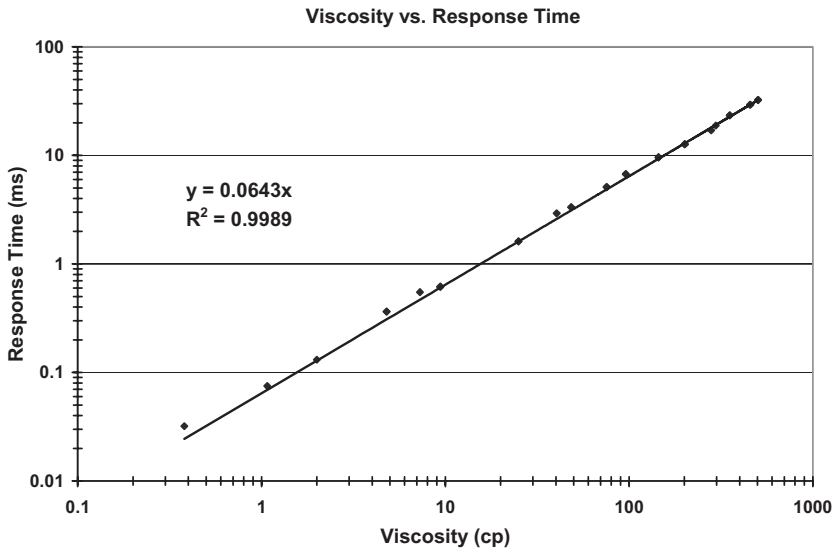

Figure 5. Viscosities of known fluids and viscosity standards plotted against measured response times.

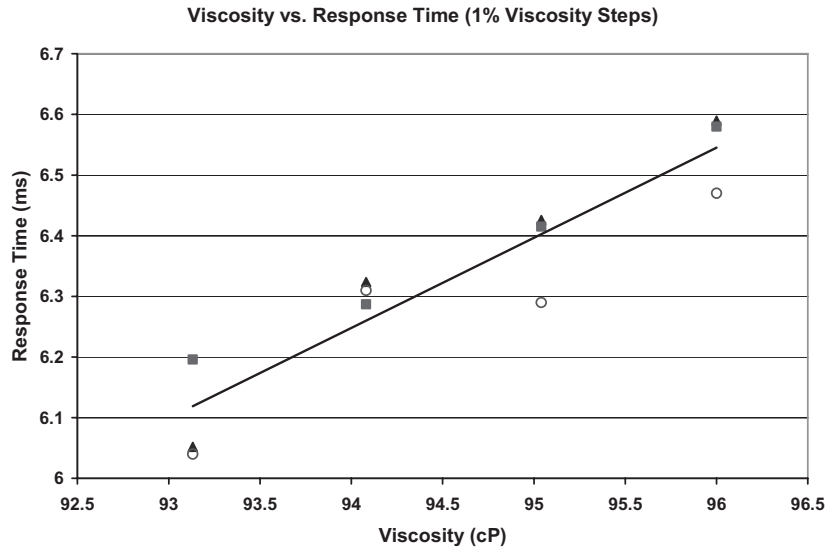

Figure 6. Three measurements made at 1\% viscosity change intervals, showing the resolution is on the order of $2 \%$.

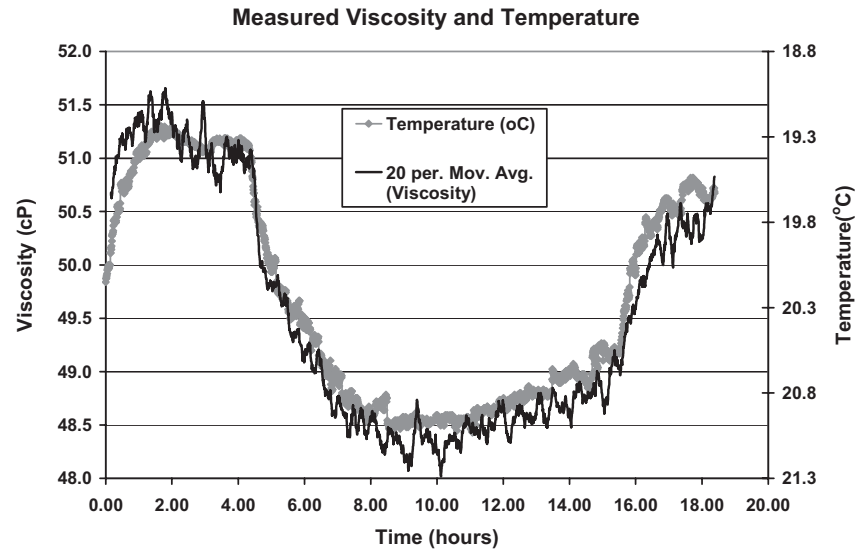

Figure 7. Viscosity and Temperature overnight monitoring. 
This change is consistent with the measured viscosity temperature dependence for the oil, which is approximately $2 \%$ per degree Celsius at $20^{\circ} \mathrm{C}$. This data indicates that the sensitivity of the device is better than the measured $2 \%$ for controlled temperature conditions, with values of $\pm 0.5 \%$ more representative.

Viscosity is a very important parameter used in the evaluation of lubricating fluids. As a lubricant degrades during operation, the viscosity monotonically increases. In order for the MEMS viscosity sensor to be viable for evaluation of degraded lubricants, it must be sensitive enough to distinguish between the viscosity of a fresh and degraded sample. To test this, a sample of Castrol 5050 was thermally degraded on a hot plate set at $250^{\circ} \mathrm{C}$ for 4 hours. The viscosity of both the fresh sample and the thermally degraded sample was then tested using the same device. As shown in figure 8, the device is sensitive enough to easily resolve the viscosity change associated with thermally degraded oil. During our experiment, the viscosity of the thermally degraded oil increased by approximately $10 \%$. Oil that degrades during machine use would likely have a larger viscosity change. Previous work has shown that the viscosity of automobile engine oil increases by approximately $40 \%$ as it degrades over useful life [8].

Response Time / Viscosity at Room Temperature Baseline Castrol 5050 and Thermally Degraded Castrol 5050

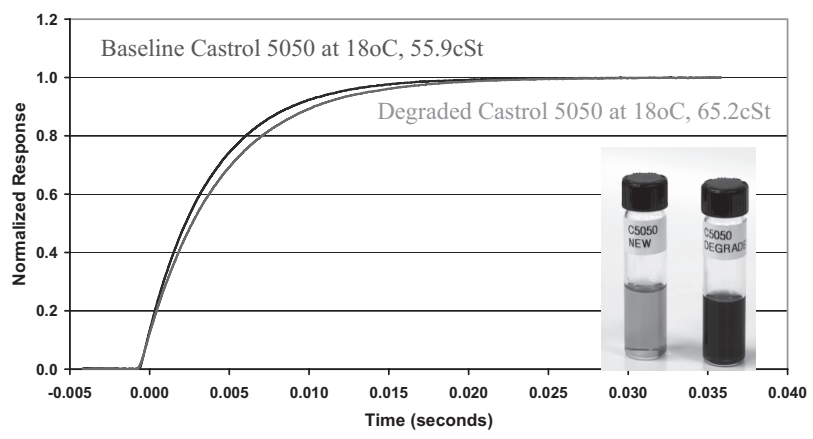

Figure 8. Response times for fresh oil and thermally degraded oil.

Most of the experiments described here were performed using fresh, clean samples with no particulates. Although the device does have moving parts with small clearances $(2 \mu \mathrm{m})$, the sensor has proven to be relatively immune to most contaminants. Many experiments in degraded oils saturated with visible particulates have been unaffected by the contaminants. However, we have seen occasional device failures when small particulates on the order of the size of the comb finger spacing have become stuck between comb teeth. Sensors deployed in dirty environments will likely need to either be placed near the in line filter or packaged with lids with integrated filters. Mesh screens with small openings ( $\sim$ um) and large open area $(\sim 50 \%)$ are commercially available.

\section{CONCLUSIONS}

A MEMS viscosity sensor was designed and fabricated. The measured performance of this device indicates that it is capable of measuring a very wide range of viscosities $(>1000 x)$ with a single device. In addition, the excellent linearity and sensitivity of the device shows that it performs comparable to large scale, commercially available viscometers. A viscosity sensor of this type facilitates real time, in-situ viscosity measurements. This is essential for applications such as lubrication health monitoring for condition-based maintenance of motorized vehicles, automation equipment, and other rotary equipment. Due to its small size $(2 \mathrm{x}$ $2 \mathrm{~mm})$ and limited fluid sampling requirement $(20 \mu \mathrm{L})$, the device can easily be mounted inside a lubrication reservoir of any piece of equipment to monitor viscosity degradation during operation.

Future work will concentrate on characterizing the temperature performance and reliability of the device. Many of the in-situ applications would require the device to operate at elevated temperatures up to $150^{\circ} \mathrm{C}$. Although the preliminary data looks promising, long-term reliability data needs to be collected to fully characterize the device. In addition, the effects of particulate contamination will be characterized, along with mitigation techniques such as using in line filters and adjusting the comb finger spacing.

\section{ACKNOWLEDGEMENTS}

This research was sponsored by Rockwell Automation. The authors thank Fred Discenzo of Rockwell Automation for his technical support on this program. Additionally, the authors thank Martin Kendig for insightful conversations regarding lubricant health monitoring. Finally, the authors thank Kathleen Garrett for all her support in fabrication of the viscosity sensors.

\section{REFERENCES}

[1] O. Brand, J M. English, S. A. Bidstrup, M. G. Allen, "Micromachined Viscosity Sensor for Real-Time Polymerization Monitoring", Technical Digest of the 1997 International Conference on Solid-State Sensors and Actuators, Chicago, IL, June 16-19, (1997), pp. 121-124.

[2] M. K. Jain, C. A. Grimes, "Effect of Surface Roughness on Liquid Property Measurements Using Mechanically Oscillating Sensors", Sensors and Actuators A, 100, (2002), pp. 63-69.

[3] B. Jakoby, M. Scherer, M. Buskies, H. Eisenschmid, "Microacoustic Viscosity Sensor for Automotive Applications", Technical Digest of the 2002 IEEE International Conference on Sensors, Orlando, FL, June 12-14, (2002), pp. 1587-1590.

[4] L. Meirovitch, "Principles and Techniques of Vibrations", Prentice-Hall, Inc., Upper Saddle River, New Jersey, NJ, (1997), pp. 110.

[5] T. L. Sounart, T. A. Michalske, K. R. Zavadil, "FrequencyDependent Electrostatic Actuation in Microfluidic MEMS", Journal of Microelectromechanical Systems, 14, 1, (2005), pp. 125-133.

[6] R. Borwick, P. Stupar, J. DeNatale, R. Anderson, C. Tsai, K. Garrett, R. Erlandson, "A High Q, Large Tuning Range MEMS Capacitor for RF Filter Systems", Sensors and Actuators A, 103, (2003), p. 33.

[7] www.brookfieldengineering.com

[8] A. Basu, A. Berndorfer, C. Buelna, J. Campbell, K. Ismail, Y. Lin, L. Rodriguez, S. Wang, "Smart Sensing of Oil Degradation and Oil Level Measurements in Gasoline Engines", Technical Digest of SAE 2000 World Congress, Detroit, MI, March 6-9, (2000), pp. 1-7. 